\title{
Optimization of Coaxial Couplers
}

\author{
Tushar K. Ghosh, Member, IEEE, Richard G. Carter, Senior Member, IEEE, \\ Antony J. Challis, Kevin George Rushbrook, and Darrin Bowler
}

\begin{abstract}
A simple and accurate method has been developed to optimize the performance of coaxial couplers for traveling-wave tubes using a 3-D electromagnetic field simulation code. The new technique, which is a combination of numerical and analytical procedures, is presented with a comparison with conventional practice. By using this method, a number of couplers with SubMiniature A, Threaded Neill-Concelman, and waveguide output for different tubes were modeled, validated by the experimental cold test data, and optimized. The use of this technique can reduce the optimization time by up to $90 \%$ in comparison with the conventional numerical only approach.
\end{abstract}

Index Terms-Coaxial couplers, impedance, optimization methods, transmission line theory, traveling-wave tube (TWT).

\section{INTRODUCTION}

$\mathbf{I}$ N ANY TRAVELING-wave tube (TWT), the impedance of a coupler should have a good match with the interaction circuit for maximum power transfer and minimum reflection from both input and output ends. Analytical methods can be used for quick prediction of the coupler impedance, but they are generally limited to simple and specific slow-wave circuits [1], [2] where equivalent transmission line theory [3] can be used in calculating the circuit impedance. If, however, the shapes of dielectric support rods and metal vanes become complex, which is quite common, the analytical methods fail to achieve sufficient accuracy. Therefore, a 3-D electromagnetic code based on numerical methods should be used to determine the impedance of the slow-wave circuit [4] to match with the assembly of the coaxial coupler and the coaxial or waveguide output. In previous attempts, integrated models of the coupler and the interaction circuit were simulated in HFSS to compute the return loss of the combined assembly [5], [6]. However, in all these cases, the computational time required for each simulation is very long, making the optimization task very costly. Sometimes, it may not even be possible to find a near-optimum solution within the stipulated time. Moreover, an integrated assembly of slow-wave structure, coupler, and waveguide or Threaded Neill-Concelman (TNC) connector is often difficult to model with reasonable accuracy because of the large number of mesh points needed. To overcome these limitations, the "split model" method, which is proposed here for the optimization of couplers, is several times faster than the conventional approach

Manuscript received February 21, 2007; revised April 18, 2007. The review of this paper was arranged by Editor W. Menninger.

T. K. Ghosh, A. J. Challis, K. G. Rushbrook, and D. Bowler are with e2v technologies, CM1 2QU Chelmsford, U.K. (e-mail: tushar.ghosh@ e2v.com).

R. G. Carter is with the Engineering Department, Lancaster University, LA1

4YR Lancaster, U.K. (e-mail: r.carter@lancaster.ac.uk).

Digital Object Identifier 10.1109/TED.2007.898045

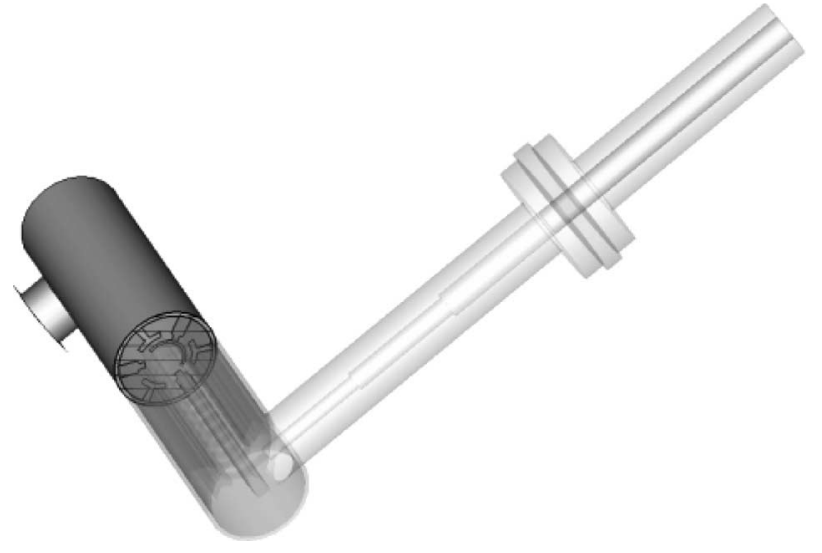

Fig. 1. Cutaway view of the integrated model of a slow-wave circuit and coaxial coupler.

and can be used with any 2-D or 3-D electromagnetic code. A comparison between the simulated results obtained using both methods and experimental results validating them is presented in this paper. To demonstrate the capability of the split model method, a computer code implementing it has been used in conjunction with the 3-D electromagnetic field simulation code Microwave Studio ${ }^{1}$ to optimize the performance of a number of couplers with Sub-Miniature A (SMA), TNC, and waveguide output.

\section{Conventional Optimization Technique}

To demonstrate the conventional optimization technique and its limitations, an integrated model of the slow-wave circuit and coupler of a 6-18-GHz TWT, as shown in Fig. 1, was simulated [7] using Microwave Studio. The model consists of several turns of helix with finite tape thickness, dielectric support rods, T-shaped metallic vanes, body tube, coaxial transition, and ceramic window assembly. The dielectric properties of the materials were defined to ensure an accurate computation of phase velocity of the wave and of the impedance of the total assembly. Perfect conducting boundary conditions were applied at the axial truncation points of the body tube [8]. Both input and output couplers were terminated with waveguide ports to simulate infinitely long waveguides at the boundaries of the calculation domain. Once the steady state was reached, S11 was calculated from the amplitude and the phase of the signals at the ports.

To optimize the performance of the coupler, the model was simulated several times with different dimensions, as shown in

\footnotetext{
${ }^{1}$ Supplied by Computer Simulation Technologies.
} 


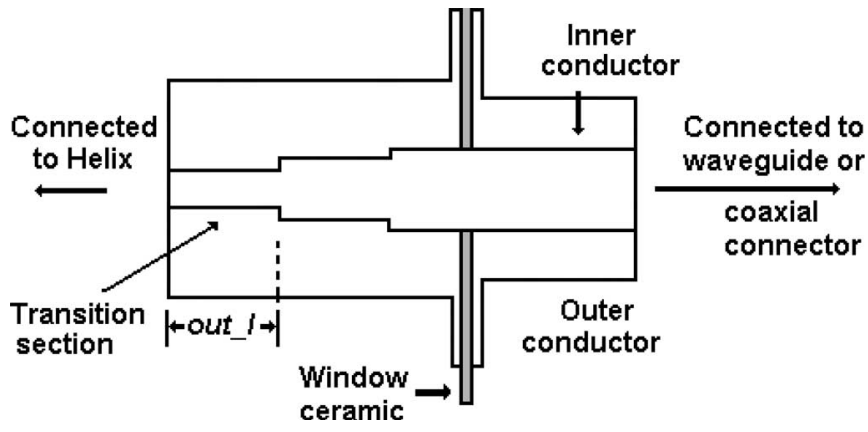

Fig. 2. Typical coaxial coupler with out_l as the length of the transition section connected to the helix.

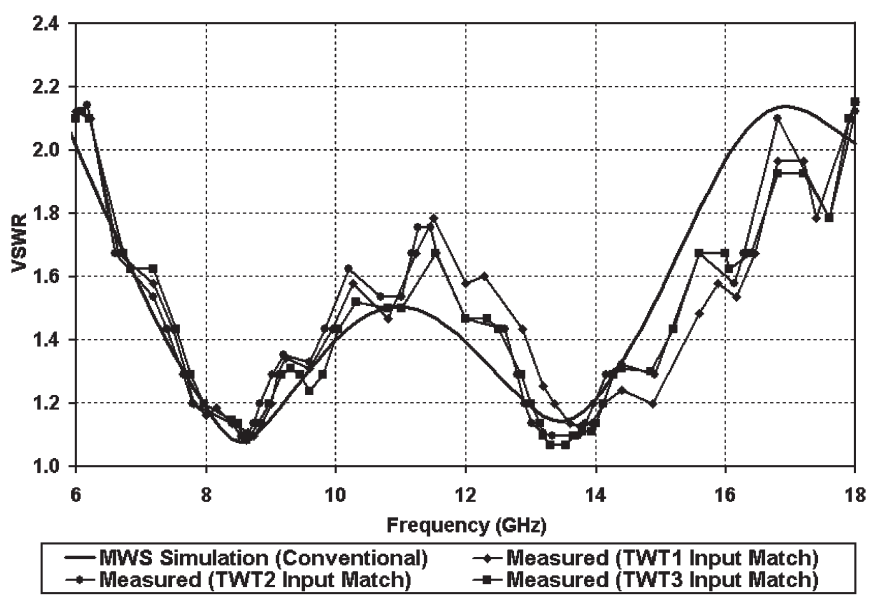

Fig. 3. Comparison between the simulated and experimental VSWR of the input coupler of a $6-18-\mathrm{GHz}$ TWT.

Fig. 2. The optimized voltage standing wave ratio (VSWR) is compared with the cold test results in Fig. 3, which shows excellent agreement. The similarity of the experimental results of the three samples of the tube shows that the tolerances set in the design were maintained during the fabrication of each tube.

For this computational model, the simulation time for each iteration was nearly $30 \mathrm{~min}$ on a PC with a $2.8-\mathrm{GHz}$ processor and 2-GB RAM. Therefore, the total computation time for optimization and sensitivity analysis to finalize the design and tolerances of a coupler is enormous as it involves hundreds of iterations. It is shown later in this paper that the performance of some optimized couplers can still be improved significantly within a short time by using the split model method.

\section{Proposed Technique}

It was observed that nearly $90 \%$ of the time taken for each simulation was due to the inclusion of the slow-wave circuit in the model. However, it remains unchanged throughout the optimization process. Moreover, because a large number of mesh points are required to model the complex shape of the helix, the available mesh points are inadequate to model the coupler with reasonable accuracy as the total number of mesh nodes is limited by the computer hardware. The split model procedure overcomes these limitations by using a combination of numerical and analytical procedures.

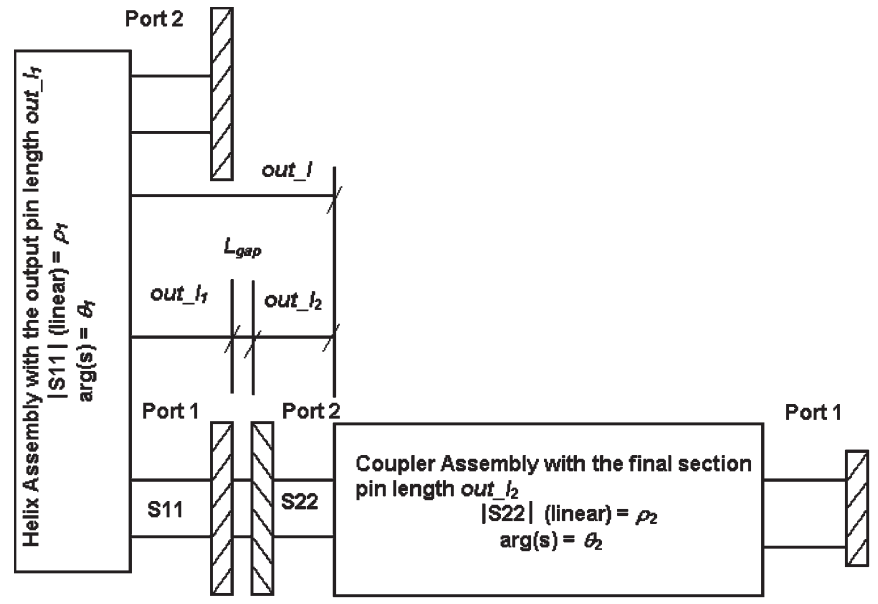

Fig. 4. Split models of helix and coupler assemblies for uniform coaxial transition section.

\section{A. Implementation}

If the slow-wave circuit is removed from the integrated model during repetitive computations, then the simulation time is reduced drastically while all the mesh nodes become available to model the coupler assembly. Therefore, we replaced the slow-wave circuit with its equivalent reflection coefficient during optimization.

The technique is illustrated in the following steps.

1) The integrated model (see Fig. 1) is split into two at the transition section of the coupler (see Fig. 2) to create the helix and the coupler assembly models, as shown in Fig. 4. The helix assembly consists of the slow-wave circuit and an output coaxial pin of arbitrary length out_ $l_{1}$, whereas the coupler assembly consists of the rest of the integrated model but with an arbitrary length of the final section equal to out_l $l_{2}$. These two lengths remain the same throughout the optimization process. It is necessary to keep these lengths short so that, together, they are less than or equal to the total length of the transition section in the final assembly.

2) A number of helix assembly models are created and simulated with different impedances at the output pin. For example, 20 helix models can be created and simulated with output pin impedances $50,55,60 \Omega, \ldots$, etc. The amplitude $\rho_{1}$ and the phase $\theta_{1}$ of S11 (see Fig. 4) corresponding to each impedance value are stored for a number of frequencies (about 100) in the operating band of the TWT. An overnight simulation in batch mode creates a data bank, and there is no need for further simulation of these models if the slow-wave circuit remains unchanged.

3) In a similar way, the same number of coupler assembly models is created by making the impedance of its final section the same as the output pin of the helix assembly. Thus, 20 pairs of helix and coupler assembly models are created where each pair corresponds to a specific impedance. The simulated values of the amplitude $\rho_{2}$ and the phase $\theta_{2}$ of S22 (see Fig. 4) are combined analytically with the S11 of the corresponding helix assembly for the same frequencies as in step 2 to calculate the overall 


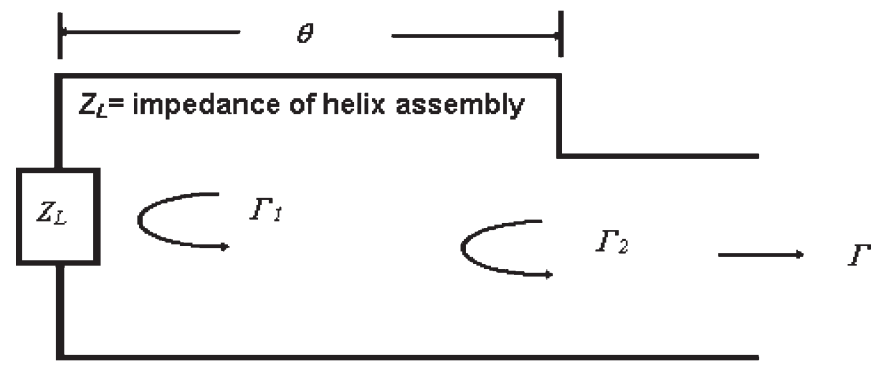

Fig. 5. Equivalent microwave circuit showing the reflection coefficient.

return loss. Details of the analytical method used for this purpose are given later in this section.

4) The performance of any pair may be optimized through a number of trials by changing the different dimensions of the coupler assembly, other than the final section, and by combining the simulated results analytically with the corresponding helix assembly. The process is repeated for other pairs, and the best match among the 20 pairs is taken as the optimum design. Further optimization can be carried out by repeating Steps 2, 3, and 4 for impedances around the best match.

The new technique has many advantages over the conventional procedure. Because the integrated model is split into two individual models, all the mesh nodes become available to model each of them with better accuracy. This also makes it possible to model simultaneously a longer slow-wave circuit and a complex coupler shape with TNC or waveguide output. It takes less than 2 min to simulate the coupler assembly with an SMA or TNC connector and $1 \mathrm{~min}$ to import the simulated results and combine them analytically. This is $10 \%$ of the time taken for the simulation of the integrated model. Therefore, the optimization can be carried out within a much shorter time.

\section{B. Combining S-Parameters (Uniform Coaxial)}

An equivalent microwave circuit of the integrated assembly is shown in Fig. 5 where $\Gamma_{1}$ and $\Gamma_{2}$ are the reflection coefficients of the helix (S11) and coupler (S22) assemblies, respectively, calculated as

$$
\begin{aligned}
& \Gamma_{1}=\rho_{1} e^{j \theta_{1}} \\
& \Gamma_{2}=\rho_{2} e^{j\left(\pi-\theta_{2}\right)}
\end{aligned}
$$

where $\rho_{1}, \theta_{1}, \rho_{2}$, and $\theta_{2}$ are all functions of frequency. S22 for the coupler assembly is in the opposite direction to S11 for the helix assembly (see Fig. 4), whereas they are in the same direction in the equivalent model. Therefore, the equivalent phase of S22 is taken as $\left(\pi-\theta_{2}\right)$ in the circuit instead of $\theta_{2}$.

A uniform coaxial line of the same impedance as the helix output pin with length $L_{\text {gap }}$ is introduced between the helix and coupler assemblies (see Fig. 4) to optimize the length of the transition section, as both out_ $l_{1}$ and out $l_{2}$ remain fixed throughout the computation. In the optimized design, the length of the transition section of the coupler out_ $l$ is equal to the total length of these three. The length $L_{\text {gap }}$ can have any positive value, including zero, and its equivalent electrical length at any frequency $f$ is

$$
\theta=2 \pi f L_{\text {gap }} / c
$$

where $c$ is the velocity of light in vacuum. The overall reflection coefficient of the combined assembly is calculated from the theory of multiple reflections [9] as

$$
\Gamma=\frac{\Gamma_{2}+\Gamma_{1} e^{-j 2 \theta}}{1+\Gamma_{1} \Gamma_{2} e^{-j 2 \theta}}
$$

where $\Gamma_{1}$ and $\Gamma_{2}$ are both complex and defined by (1) and (2), respectively. After simplification of this equation, $\Gamma$ is expressed in the form of real and imaginary parts from which its amplitude is calculated (see the Appendix). The overall VSWR over the frequency band is calculated using

$$
\operatorname{VSWR}=\frac{1+|\Gamma|}{1-|\Gamma|}
$$

\section{Combining S-Parameters (Tapered Coaxial)}

To create two models, the tapered transition section, like uniform coaxial line, is divided into two, as shown in Fig. 6. A coaxial section of small length is added at the end of the tapered line to simulate a waveguide port at the truncation point. To combine the $S$-parameters, the phase of the signals must be calculated at points $H_{2}$ and $C_{2}$, whereas the simulated values $\theta_{1 H}$ and $\theta_{2 C}$ are obtained at points $H_{1}$ and $C_{1}$ for the helix and coupler assemblies, respectively. As the amplitude of the signal remains unchanged between points on a uniform coaxial line, it is only necessary to recalculate the phase as

$$
\begin{aligned}
& \theta_{1}=\theta_{1 H}+4 \pi f T_{\mathrm{LH}} / c \\
& \theta_{2}=\theta_{2 C}+4 \pi f T_{\mathrm{LC}} / c
\end{aligned}
$$

where $T_{\mathrm{LH}}$ and $T_{\mathrm{LC}}$ are the lengths of small coaxial sections added to the helix and coupler assemblies, respectively. The VSWR of the combined assembly is now calculated using (1) $-(5)$ and setting $L_{\text {gap }}=0$.

\section{Computer Code}

A computer code "COUPLED" implementing the analytical method was developed in $\mathrm{C}$ and runs in DOS. For any impedance, this program takes data files of the amplitude versus frequency and phase versus frequency for both assemblies, and the value of $L_{\text {gap }}$ as input to calculate the overall return loss and VSWR for the frequency band of operation. An interpolation routine was introduced to make sure that the amplitude and phase values of the assemblies correspond to the same frequencies.

\section{E. Comparison}

Two couplers with transition sections of different shapes were simulated using both the conventional and the new 
Port 2

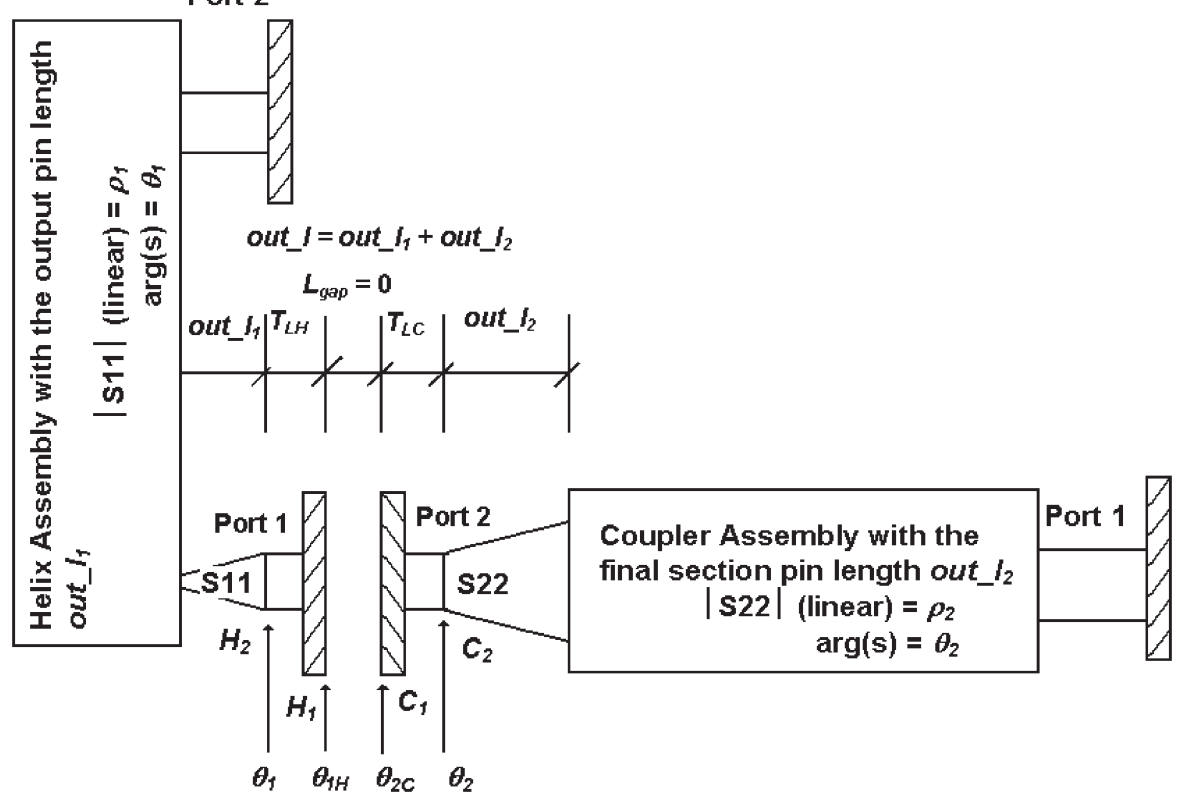

Fig. 6. Split models of helix and coupler assemblies for tapered coaxial transition section.

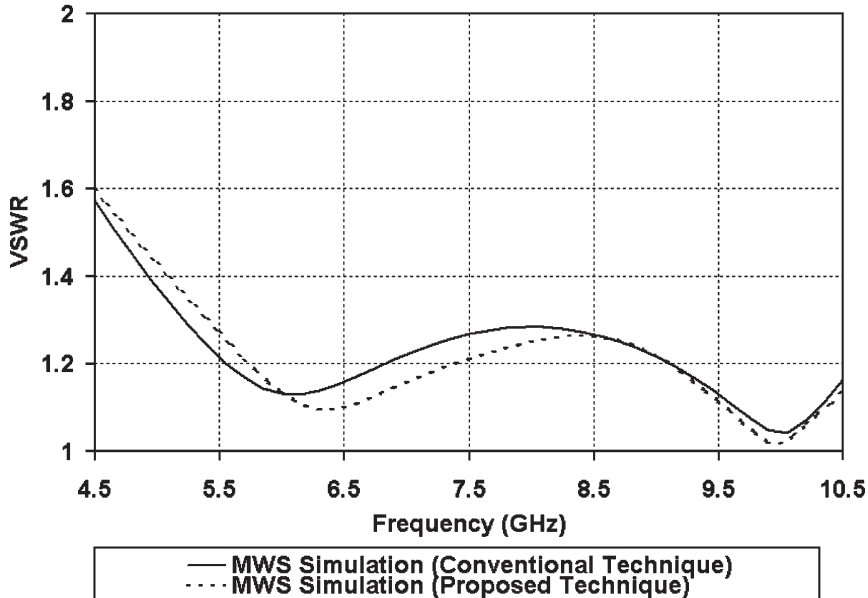

Fig. 7. Comparison between the simulated VSWR of the coupler with uniform coaxial transition section used in a $4.5-10-\mathrm{GHz}$ TWT.

techniques, and the results were compared to test the effectiveness of the split model method. Both couplers were fitted with SMA connectors and used in a $4.5-10-\mathrm{GHz}$ TWT. The coupler with a uniform transition section was first optimized using the split model technique and, later, was simulated using the conventional method. Good agreement between the two methods is shown in the VSWR versus frequency plot in Fig. 7. It is also observed from the plot that the coupler is optimized to have its best performance at the higher end of the frequency band, as the output power is lower in this region.

Again, the simulated results of a coupler with a tapered transition section, shown in Fig. 8, match each other closely throughout the band of operation of the TWT. Similar agreements were observed by changing the different dimensions of both coupler geometries [10]. This establishes the consistency of the proposed method.

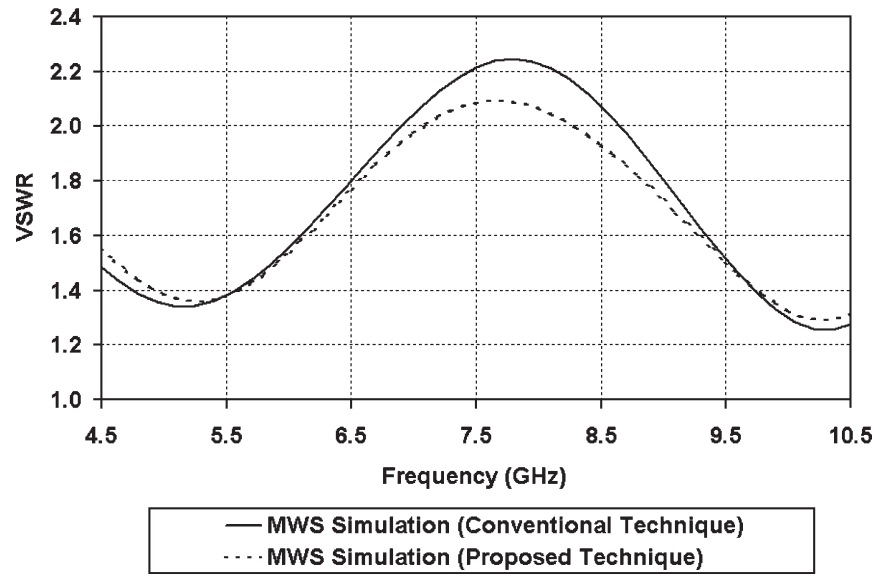

Fig. 8. Comparison between the simulated VSWR of the coupler with tapered coaxial transition section used in a $4.5-10-\mathrm{GHz}$ TWT.

\section{VALIDATION AND OPTIMIZATION}

To demonstrate the capability of the proposed method, models of three different coupler geometries with either a coaxial connector or waveguide as output were validated by cold test data and optimized. All of these couplers were originally optimized using either the conventional technique or through repetitive experimental evaluation. Optimized geometries were taken as the starting point for further optimization using the proposed method. None of the simulations included the effect of loss due to the coating on the rods.

The input coupler of a $6-18-\mathrm{GHz}$ TWT with an SMA connector was taken as the first geometry for modeling. The simulated result is compared with the cold test data in Fig. 9. Excellent agreement, validating the model, is achieved over the entire frequency band. A comparison between the original and the optimized performances in the plot reveals that, while the conventional technique was able to achieve a VSWR better 


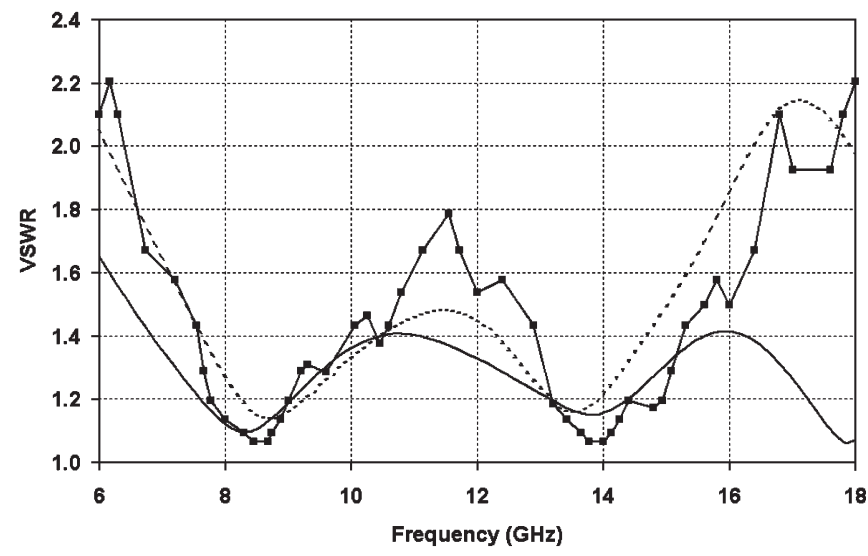

$\rightarrow-$ Measured (TWT4 Input Match) $\quad$-... Proposed Method (Original) - Proposed Method (Optimized)

Fig. 9. Comparison between the simulated and experimental VSWR of the input coupler used in a $6-18-\mathrm{GHz}$ TWT.

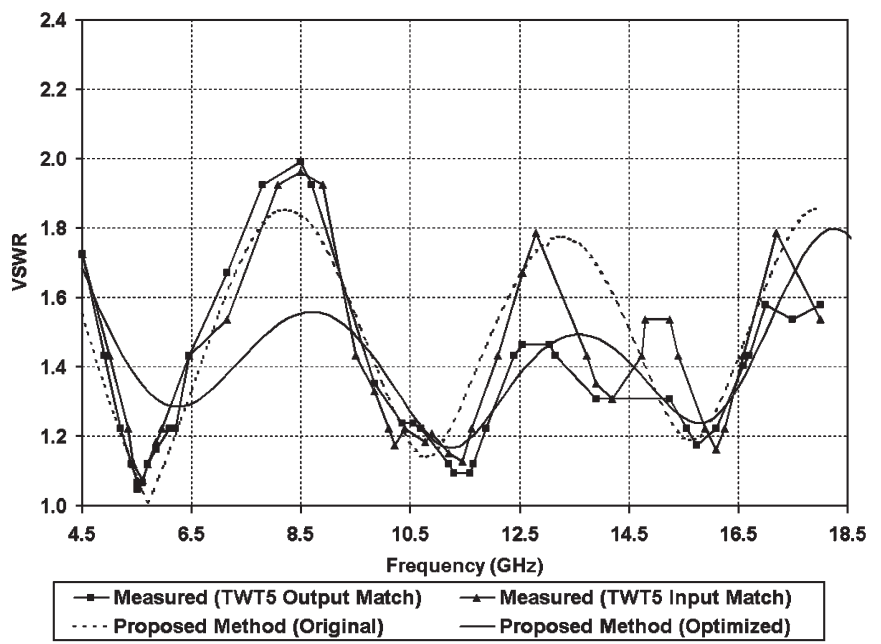

Fig. 10. Comparison between the simulated and experimental VSWR of the couplers used in a $4.5-18-\mathrm{GHz}$ TWT.

than 2.15, further optimization using the split model method improved this value to 1.65 with a significant improvement near both edges of the frequency band. It was not possible to continue the optimization using the standard technique due to time constraints.

The second geometry is a coupler with a TNC connector, used as both the input and output of a $4.5-18-\mathrm{GHz}$ TWT. An integrated model of the slow-wave circuit, the coupler with tapered transition section, and connector could not be created with reasonable accuracy due to its size. Simulated results for both the original and optimized geometries, which are obtained using the split model method, are compared with the cold test data in Fig. 10. The close match between experimental and simulated results for the original geometry validates the model. It is also observed that the original VSWR is better than 1.9 with the worst values around 8.5-GHz. Optimization was able to improve this significantly in the middle of the band with a little deterioration at the lower end of the band.

The third coupler uses a waveguide as the output of the 6-18-GHz TWT. Like the previous case, the integrated geom-

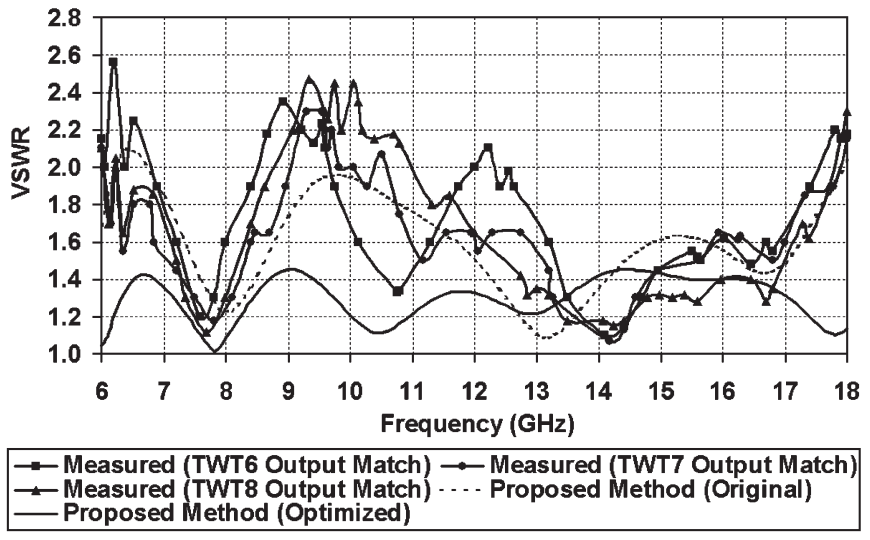

Fig. 11. Comparison between the simulated and experimental VSWR of the output couplers used in a $6-18-\mathrm{GHz}$ TWT.

etry could not be modeled using the conventional technique because it required too many mesh nodes. The simulated VSWR plotted in Fig. 11 closely resembles the three sets of cold test data, although there is a slight difference. In practice, the waveguide has a number of screws to fine-tune the overall performance according to the requirement of the output power, e.g., a lower VSWR may be desirable at the higher frequencies with a compromise elsewhere in the band. The positions of the screws inside the waveguide at the tuned condition vary from one tube to another and were not known. Hence, the tuning screws were not included in the model, which may be the reason for slight difference in the results. The optimized VSWR of the coupler is better than 1.5 , with a significant improvement throughout most of the band.

\section{CONCLUSiON}

A new method for modeling and optimizing coaxial couplers has been described and validated by experimental results. It was shown that this method could be used to reduce the time for one design iteration by $90 \%$ in comparison with the conventional procedure. Using this technique, significant improvements were achieved in the performance of couplers, which had originally been optimized by either the conventional procedure or by repetitive experimentation. The advantages of the technique were demonstrated by optimizing the couplers with TNC and waveguide outputs, both of which could not be modeled with reasonable accuracy using the conventional method because of the limitation in the number of mesh nodes handled by the computer hardware. Thus, the proposed method can be used as a fast and accurate way of modeling and optimizing large geometries. Future work will include the effect of loss due to the coating on the rods, which may improve the match.

\section{APPENDIX}

The reflection coefficient parameters in (1) and (2) can be expressed as

$$
\begin{aligned}
& \Gamma_{1}=P+j Q \\
& \Gamma_{2}=R+j S
\end{aligned}
$$


where

$$
\begin{aligned}
& P=\rho_{1} \cos \theta_{1} \\
& Q=\rho_{1} \sin \theta_{1} \\
& R=\rho_{2} \cos \left(\pi-\theta_{2}\right) \\
& S=\rho_{2} \sin \left(\pi-\theta_{2}\right) .
\end{aligned}
$$

Now, the combined reflection coefficient in (4) can be expressed as

$$
\Gamma=\frac{A+j B}{C+j D}
$$

where

$$
\begin{aligned}
A= & R+P \cos 2 \theta+Q \sin 2 \theta \\
B= & S+Q \cos 2 \theta-P \sin 2 \theta \\
C= & 1+R(P \cos 2 \theta+Q \sin 2 \theta) \\
& +S(P \sin 2 \theta-Q \cos 2 \theta) \\
D= & S(P \cos 2 \theta+Q \sin 2 \theta) \\
& -R(P \sin 2 \theta-Q \cos 2 \theta) .
\end{aligned}
$$

Further simplification of (A3) can be made to express $\Gamma$ in terms of real and imaginary parts as

$$
\Gamma=\frac{A C+B D}{C^{2}+D^{2}}+j \frac{B C-A D}{C^{2}+D^{2}} .
$$

\section{ACKNOWLEDGMENT}

The authors would like to thank the Directors, e2v technologies (U.K.), for their support and for granting permission to publish this paper.

\section{REFERENCES}

[1] B. Jia, C.-W. Baik, and G.-S. Park, "The design of the input and output transformer for wideband helix TWT," in Proc. 2nd Int. Conf. Microw. and Millimeter Wave Technol., Beijing, China, 2000, pp. 715-719.

[2] A. K. Sinha, V. V. P. Singh, V. Srivastava, and S. N. Joshi, "On the design of coaxial coupler having multi-section short transformer for compact sized power helix traveling wave tubes," in Proc. Int. Vac. Electron. Conf., Monterey, CA, 2000, pp. p2.25-p2.26.

[3] B. N. Basu, Electromagnetic Theory and Applications in Beam-Wave Electronics. Singapore: World Scientific, 1996.

[4] C. L. Kory and J. A. Dayton, Jr., "Accurate cold-test model of helical TWT slow-wave circuits," IEEE Trans. Electron Devices, vol. 45, no. 4, pp. 966-971, Apr. 1998.

[5] A. K. Agarwal, S. Raina, and L. Kumar, "A novel approach for simulation of coaxial coupler for helix TWTs using HFSS," in Proc. 4th IEEE Int. Vacuum Electron. Conf., Monterey, CA, 2001, pp. 58-59.

[6] M. K. Alaria, P. Dubey, A. K. Sinha, V. V. P. Singh, and V. Srivastava, "Improved approach of designing output coupler for Ku-band space helix traveling wave tube," in Proc. APMC, New Delhi, India, 2004. CDROM.

[7] M. Duffield, "Establishing a technique for designing the RF connectors used on helix travelling wave tubes," M.S. thesis, Dept. Phys., Univ. Strathclyde, Glasgow, U.K., 2006.

[8] F. Kantrowitz and I. Tammaru, "Three-dimensional simulations of frequency-phase measurements of arbitrary coupled-cavity RF circuits," IEEE Trans. Electron Devices, vol. 35, no. 11, pp. 2018-2026, Nov. 1988.

[9] R. E. Collin, Foundations for Microwave Engineering. New York: IEEE Press, 2000.

[10] T. K. Ghosh, A Novel Technique for Design Optimization of Couplers. Chelmsford, U.K.: e2v Technologies, 2005.

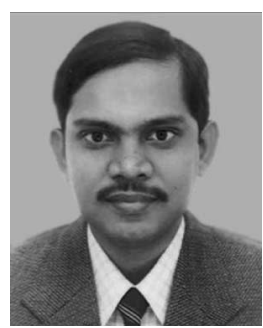

Tushar K. Ghosh (M'00) received the B.E. degree from Bengal Engineering and Science University (formerly, Bengal Engineering College, Calcutta University), Shibpur, India, in 1990, and the M.E. degree from Jadavpur University, Kolkata, India, in 1993, both in electronics and telecommunication engineering, and the Ph.D. degree in engineering from Lancaster University, Lancaster, U.K., in 2002, for his work on 3-D modeling and optimization of multistage collectors.

From 1993 to 1994, he worked on electromagnetic pulse sensors as a Senior Research Fellow with Jadavpur University. In 1994, he joined the Central Electronics Engineering Research Institute (CEERI), Pilani, India, as a Project Scientist and was a Scientist from 1995 to 2004. During this period, he was a key member of the C-band and Ku-band space traveling-wave tube (TWT) project teams where he was involved in the design and development activities. He has developed a number of computer codes implementing advanced optimization techniques, which are currently being used at CEERI for the design purpose. In 2004, he joined e2v technologies Ltd., Chelmsford, U.K., as a Principal Engineer, where he is currently involved in improving the design and modeling techniques of TWTs. His primary research interests are in vacuum device physics, modeling, and design. He has authored or coauthored a number of papers in journals and conferences.

Dr. Ghosh was awarded the Commonwealth Scholarship from 1999 to 2002. He was one of the first three recipients of the IEEE-EDS Graduate Student Fellowship award in 2001.

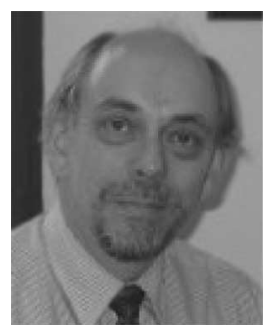

Richard G. Carter (M'97-SM'01) received the degree in physics from the University of Cambridge, Cambridge, U.K., in 1965, and the Ph.D. degree in electronic engineering from the University of Wales, Cardiff, U.K., in 1968, for his work on the propagation of waves on neutralized ion beams.

From 1968 to 1972, he was with English Electric Valve Company, Ltd., as a Development Engineer, where he worked on high-power traveling-wave tubes. In 1972, he joined the Engineering Department of the University of Lancaster, Lancaster, U.K., as a Lecturer and was promoted to Senior Lecturer in 1986 and Professor of electronic engineering in 1996. His research interests include electromagnetics and microwave engineering with particular reference to the theory, design, and computer modeling of microwave tubes and particle accelerators.

Prof. Carter is a fellow of the IET and a member of the Vacuum Electronics and Compact Modelling Technical Committees of the IEEE Electron Devices Society.

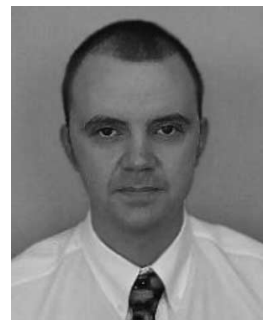

Antony J. Challis received the $\mathrm{HNC}$ in electromechanical engineering from Anglia Polytechnic University (APU), Chelmsford, U.K., in 1987.

$\mathrm{He}$ joined e2v technologies Ltd., Chelmsford, U.K. in 1983. In 1987, he completed his Mechanical Engineering apprenticeship and became an Engineer working on helix traveling-wave tube (TWT) production support. In 1988, he joined a team of development engineers within e2v, developing new products and reengineering existing devices. With a strong background in mechanical engineering and experience gained from general vacuum technology, he progressed to Technical Authority for helix TWTs. Achievements in electron gun and PPM stack design lead to his involvement in the successful development of a range of "mini TWTs" designed for airborne decoy applications. With his knowledge on TWT design and manufacture, allied with an appreciation for the vacuum electronics business, he was promoted in 2006 as a Product Manager for TWTs and microwave amplifier systems. 


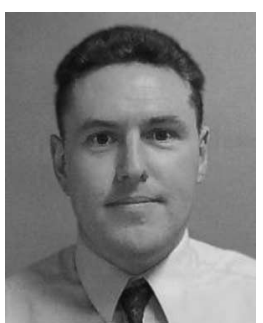

Kevin George Rushbrook received the degree from Anglia Polytechnic University (APU), Chelmsford, U.K., in 1993.

In 1988, he joined e2v technologies Ltd., Chelmsford, U.K., where he was involved in the testing of military airborne helix traveling-wave tubes (TWTs). From 1997 to 2000, he worked in the Mini TWT development program. Following a short sabbatical, he rejoined the company in 2000 . Since then, he has been working on helix TWT development projects as the Head of testing and contributing to the computer modeling team. His research interests include the design and computer modeling of helix TWTs.

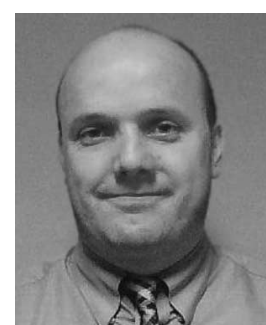

Darrin Bowler received the degree in physics from the University of Warwick, Coventry, U.K., in 1989.

In 1990, he joined e2v technologies Ltd., Chelmsford, U.K. Following a short placement in broadcast klystron and IOT engineering, he joined the program to develop high-power klystrons for particle accelerator applications. He progressed to Head of function with responsibility for the design of new klystrons including devices from 1-MW CW at $700 \mathrm{MHz}$ to $2.5 \mathrm{MW}$ at $402 \mathrm{MHz}$. Since then, he has held a number of Engineering Group Leader positions in microwave tubes and helix traveling-wave tubes (TWTs) providing both production and design and development support. He is currently the Engineering Manager of power tubes and systems at e2v. 